\title{
MEAN VS. LIFE-LIMITING FATIGUE BEHAVIOR OF A NICKEL-BASED SUPERALLOY
}

\author{
S. K. Jha ${ }^{1}$, M. J. Caton, and J. M. Larsen \\ US Air Force Research Laboratory, AFRL/RXLMN, Wright-Patterson Air Force Base, OH 45433 \\ ${ }^{1}$ Universal Technology Corporation, 1270 N. Fairfield Rd., Dayton, OH 45432
}

Keywords: Probabilistic life prediction, mean vs. life-limiting fatigue, IN100, small crack growth, crack initiation size, dwell fatigue

\begin{abstract}
The mean and the life-limiting behavior under fatigue of the nickel-based superalloy, IN100, separated (or converged) as a function of stress level and dwell loading. This behavior was related to the control of the life-limiting behavior by the smallcrack growth regime, producing its much slower response to stress level and dwell-time, relative to the mean-lifetime behavior. The lifetime probability density is therefore, modeled as a superposition of the crack growth lifetime density and a meanlifetime density. The crack growth density is calculated with the help of small-crack growth data and the distribution in the crack initiation size. The mean-lifetime density is estimated from a relatively small number of total lifetime fatigue tests. In IN100, we apply this approach to predict the effects of stress level and dwell time on the lifetime distribution and the B0.1 (1 in 1000 probability of failure) lifetime limit.
\end{abstract}

\section{Introduction}

Accurate life prediction of fracture critical components is essential for safe and reliable operation, as well as for cost effective life cycle management [1]. A probabilistic lifing method allows for the incorporation of material and microstructural uncertainties, and service-related variability in loading, environment, inspection, etc. into the life-prediction model. Often, in the past, the approach has been to empirically determine the uncertainty factors that would represent the variability in otherwise deterministically calculated lifetime [2, 3]. Recently, the emphasis has increasingly been on physics-based probabilistic life prediction methods, where the lifetime is explicitly modeled in terms of the uncertainty in the intrinsic material factors and the extrinsic variables $[4,5]$. However, the focus has been on the mean-fatigue relationship to these variables and the probabilistic treatment is accomplished by randomizing the parameters in such relationships $[5,6]$. In other words, the lifetime variability is considered as due to random deviations from the mean behavior and hence, the lower-tail is derived by extrapolating the deviations about the mean. The "mean-based" approach to fatigue variability may, in some cases, produce inconsistent probabilistic lifetime-limits with respect to material and extrinsic variables necessitating a large number of experiments for verification, as illustrated in $[7,8]$.

Another approach to probabilistic life-prediction could be to consider, that in any material the microstructure can randomly arrange into local configurations [9, 10]. These configurations can vary in terms of their size scale and/or the degree of heterogeneous deformation upon fatigue loading, and will occur with differing probabilities. Then, the total lifetime variability can be described as a probabilistic realization of sequential failure mechanisms occurring in the order of decreasing heterogeneity level [9]. Theoretically, under any nominal microstructure and fatigue loading, it is possible to assign a probability to the occurrence of a microstructural configuration where crack initiates almost instantaneously. The lower-tail behavior in this approach is therefore, limited by the (small + long) crack growth regime. This hypothesis also implies that, for a given crack initiation location (surface or subsurface), the mean-lifetimedominating mechanisms are associated with relatively lower heterogeneity levels and bear a larger role of the crack initiation regime. The mean-behavior, therefore, is considered to respond differently than the lower-tail behavior, to the microstructure and loading variables. For example in Ti-6Al-2Sn-44Zr-6Mo (Ti-6-2$4-6)$, an $\alpha+\beta$ titanium alloy, the crack initiation regime is significantly more sensitive to stress level and temperature when compared to the crack growth rates. This was shown to produce an increased separation between the mean-lifetime and the lifelimiting behavior with a decrease in either the stress level or the temperature [7].

In this paper, the above approach is applied to describing the fatigue variability behavior of the nickel-based superalloy, IN100. The effects of stress level and dwell time on the mean vs. the lifelimiting behavior are evaluated. The lifetime probability density and the B0.1 lifetime limit are predicted as a function of stress level and dwell time, with the help of small-crack growth data and a relatively small number of total lifetime experiments.

\section{Material and Experimental Procedure}

\section{Material}

The material in this study was a fine-grained, subsolvus powderprocessed nickel-based superalloy, IN100. The median $\gamma$-grain size was about $4 \mu \mathrm{m}$. The microstructure of the alloy is presented in Fig. 1 showing the $\gamma$-primary- $\gamma^{\prime}$ structure (Fig. 1(a)) and the secondary $\gamma^{\prime}$ precipitates (Fig. 1(b)).

\section{Experimental Procedure}

The specimens for the stress-lifetime (S-N) fatigue and the small crack growth tests were extracted by electro-discharge machining in the circumferential orientation from a pancake forging of the material. The specimens had cylindrical gage with button-head ends. The gage length was $15.2 \mathrm{~mm}$ and the diameter was $5 \mathrm{~mm}$. The gripping assembly was designed to extend into the furnace and was held at temperature during the elevated temperature tests. The S-N fatigue specimens were run in the low stress ground surface condition.

The fatigue experiments were conducted in load control using an MTS servo-hydraulic test system with a 458 controller. The test temperature was $650^{\circ} \mathrm{C}$. An ATS dual-zone resistance-heated furnace was used for this purpose. Thermocouple leads were welded to the ends of the specimen gage to control and monitor the temperature during the test. The frequency of $0.33 \mathrm{~Hz}$ and the stress ratio of 0.05 were employed. The dwell fatigue tests were 
run with 6 seconds dwell at the peak load while maintaining the other experimental conditions the same.

The small crack growth experiments were run in the same test system under no-dwell and 6 seconds dwell conditions, and employed both the round-bar and, in some cases, square crosssection specimens. An electropolished surface condition was used for these experiments. These involved monitoring the development and growth of multiple cracks in the same specimen throughout its lifetime. The acetate replication technique was employed to record crack lengths at predetermined intervals. At each replication step, the test was interrupted, and the specimen was unloaded and cooled down to room temperature. The replication was done under a hold load of $60 \%$ of the peak load in the fatigue cycle.
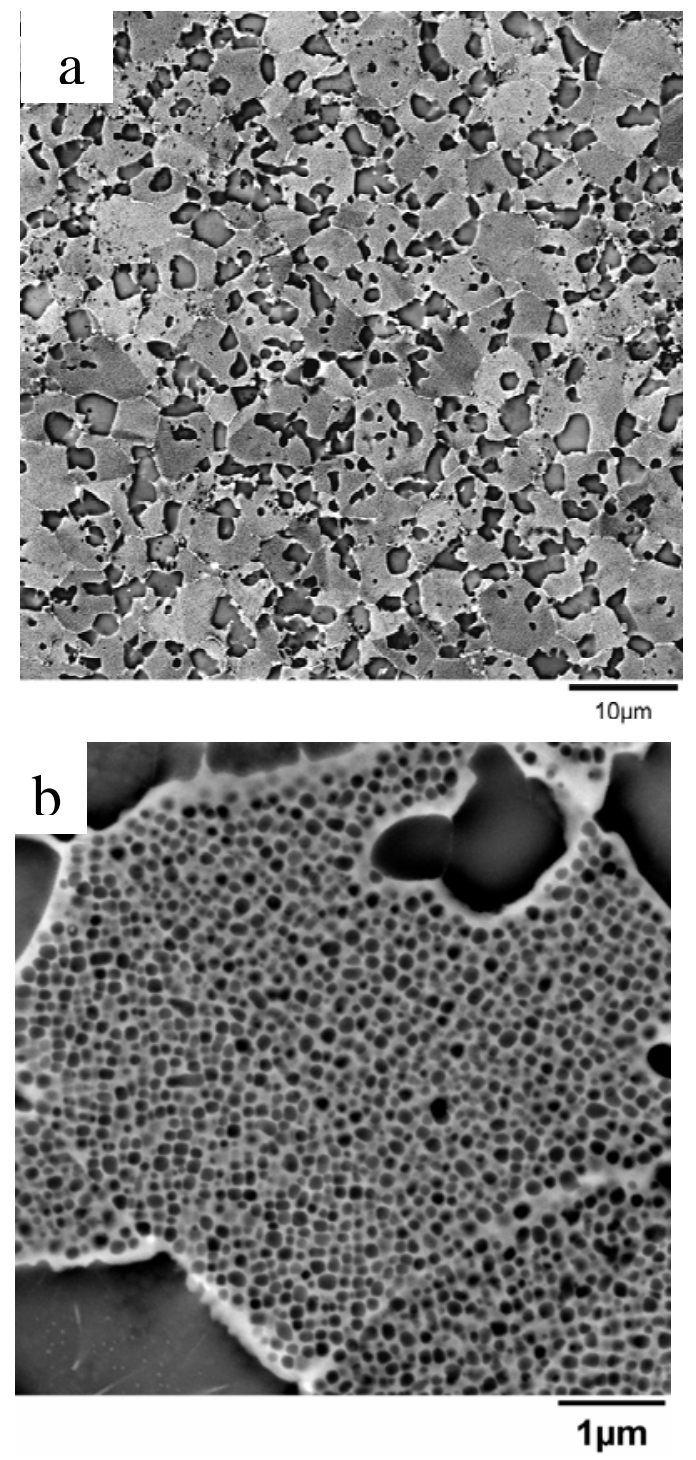

Figure 1. Microstructure of IN100; (a) the $\gamma$-primary- $\gamma^{\prime}$ morphology and (b) the secondary $\gamma^{\prime}$ structure.

The microstructure and the fatigue fracture surfaces were characterized using a Leica field emission scanning electron microscope (SEM). The crack initiation size was measured on the fracture surfaces using the ImagePro ${ }^{\mathrm{TM}}$ image analysis program. The crack length measurement on the replicas was done in an Olympus optical microscope.

\section{Results and Discussion}

Description of fatigue variability and the role of small crack growth

The fatigue variability behavior of IN100 under constant amplitude loading at $650^{\circ} \mathrm{C}$ is shown in Fig. 2. The mean vs. lifelimiting behavior is illustrated in Fig. 2(a) and the cumulative distribution functions (CDF) are plotted in Fig. 2(b). Figure 2(a) indicates that a separation of lifetimes occurred with a decrease in the stress level causing the mean-lifetime, represented by the dashed line, to diverge from the life-limiting response. Such a separation could occur when, relative to the mean-dominating behavior, the life-limiting behavior has a much slower response to stress level. Besides the stress level, the mean and the life-limiting behavior can be affected to different degrees as a function microstructure and temperature producing separation / overlap between the two as shown in [7].

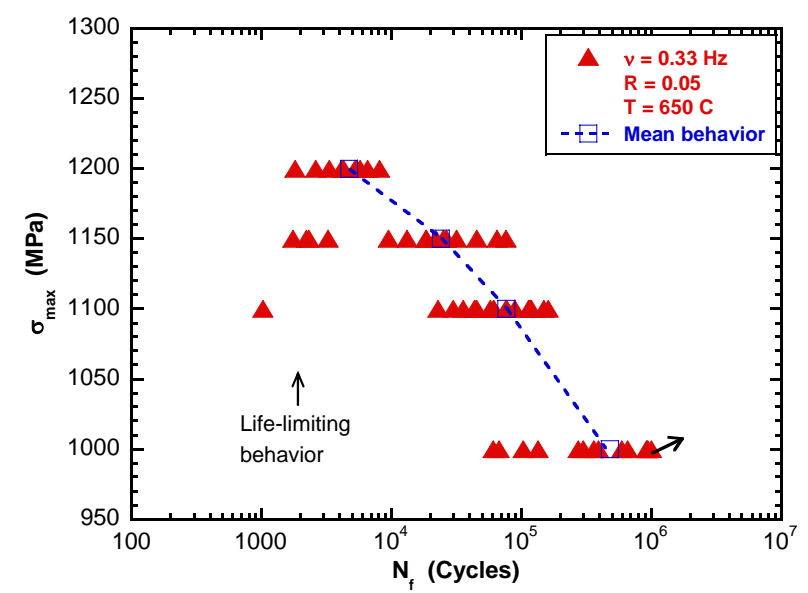

(a)

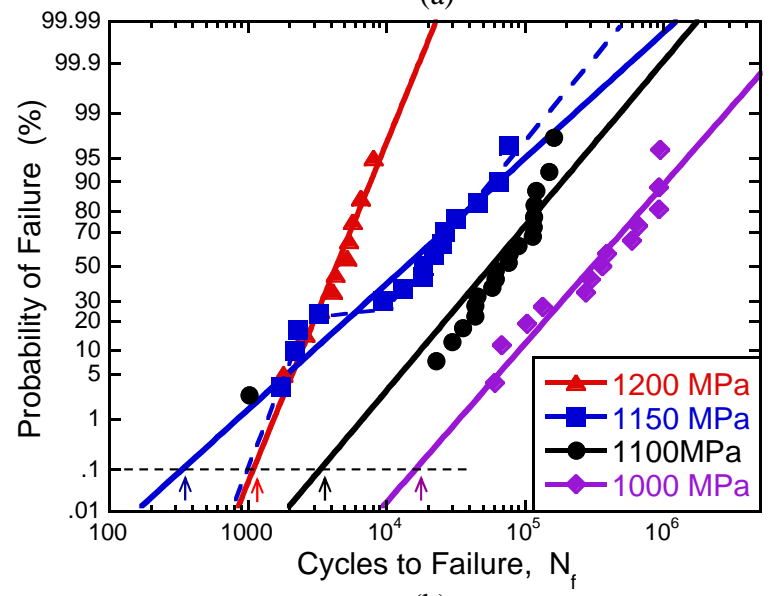

(b)

Figure 2. The fatigue variability behavior of IN100; (a) The mean vs. life-limiting behavior and (b) Cumulative distribution functions showing the B0.1 lifetimes. 
Figure 2(b) shows that the B0.1 lifetimes (indicated by arrows) based on the extrapolation of total variability to the lower tail do not exhibit a consistent trend with respect to stress level. For example, a significantly higher B0.1 lifetime is predicted at 1200 $\mathrm{MPa}$ than at $1150 \mathrm{MPa}$. On the other hand, although the predicted B0.1 lifetime at $1100 \mathrm{MPa}$ is larger than at $1150 \mathrm{MPa}$, it is nonconservative (i.e., larger) with respect to the observed minimum lifetime at that stress level. Such anomalous trends may be attributable to the assumption that the increase in uncertainty with respect to stress level (or any other variable) can be described as an increase in the deviation from the mean behavior. Further, it is noted that, while in some cases, the separation of lifetimes may not be discernible; in others it may require an impractical number of experiments to observe the same. In the following sections we discuss, perhaps, a more accurate representation of the lower tail and the total lifetime probability density, which provides physically consistent probabilistic lower lifetime-limits, independent of the number of total-lifetime experiments.

An examination of the crack initiation modes revealed that the life-limiting distribution, at lower stress levels, consisted of surface non-metallic particle initiated failures while the meandominating distribution consisted of a mixture of subsurface NMP-initiated and surface pore-initiated failures. This is summarized in Fig. 3 in terms of the crack initiation size vs. lifetime relationship. The sizes reported in Fig. 3 were measured in fracture surfaces. Figure 3 shows that there is a slight tendency towards larger NMP-related crack initiation sizes at $1100 \mathrm{MPa}$ than at $1150 \mathrm{MPa}$. However, the separation of lifetimes is governed by the mode and the location of crack initiation rather than the size.

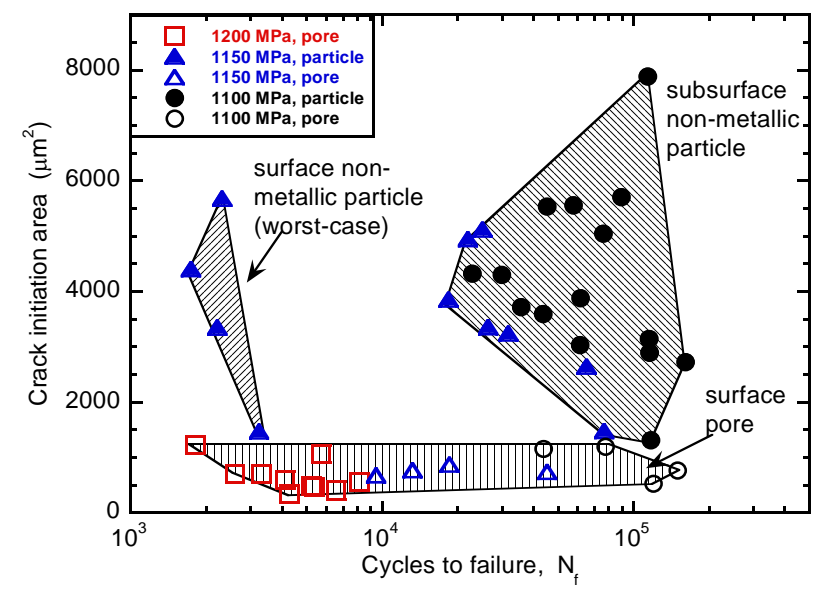

Figure 3. The separation of lifetimes with respect to crack initiation mechanisms in IN100.

The Role of the small crack growth regime. As stated previously, the separation of mean-lifetime behavior from the life-limiting response with decreasing stress level can be attributed to the relatively stronger sensitivity of the mean-behavior to stress level. In other materials, including Ti-6-2-4-6 [7, 9, 10] and a $\gamma$-TiAl based alloy [8], we have shown that this slower response of the life-limiting behavior to stress level is due to its dominance by the crack growth regime. Physically, this was reasoned as due to the development of a hierarchy of heterogeneity levels at any given loading condition producing a sequential selection of failure mechanisms in the order of decreasing heterogeneity level [9]. A probability can, therefore, be assigned to the occurrence of a purely crack growth lifetime controlled mechanism, irrespective of the nominal microstructure and loading condition [9]. The variability in the crack growth behavior, therefore, is a critical input in describing the lower tail of the lifetime probability density.

By some definitions, the small-crack growth represents the regime where the crack front spans only a few microstructural units and therefore, is strongly influenced by the local variation in properties and microstructural boundaries. Small cracks are known to grow below the threshold for long cracks, and have faster rates at the same stress intensity factor range $(\Delta K)$. The strong microstructure-scale influence accentuates as larger degree of fluctuation in growth rate as well as increased variability across different small-cracks in the same sample, when compared to the long crack. Of course, part of the fluctuation in small-crack growth rates can also be attributed to the frequency of data collection and the data reduction technique. Therefore, for effective comparison, similar measurement intervals and data reduction technique need to be employed across all cracks. Here, the crack growth rates were calculated by using the three-point sliding polynomial fit to the crack depth $(a)$ vs. cycle $(\mathrm{N})$ data. The stress intensity factor was calculated using the Foreman and Shivakumar K-solution for an elliptical surface crack in a round bar [11]. In addition to the round-bar geometry, some of the small crack growth data shown in Fig. 4 were obtained from square cross-section samples but with similar cross-sectional area and gage length as the cylindrical specimens. The K-solution for elliptical surface crack by Newman and Raju [12] were used for crack growth analysis in this geometry.

The variability in the small crack growth rates in IN100 at $650^{\circ} \mathrm{C}$ is compared to the long crack growth regime in Fig. 4. Figure 4(a) compares the growth rates of the NMP and the void initiated cracks and Fig. 4(b) shows the effect of stress level on the small crack growth behavior. These figures indicate that the long crack growth behavior showed a significantly less degree of variability than the small crack regime. Furthermore, the variability in the small crack growth behavior (for void-initiated cracks at 1150 $\mathrm{MPa}$ ) reduced to a similar level as in the long crack beyond the crack length of about $100 \mu \mathrm{m}$. Clearly, the NMP-initiated small cracks showed higher growth rates than the pore-initiated cracks (Fig. 4(a)). Besides the relatively larger crack initiation sizes associated with the NMP (Fig. 3), this could also be due to the tensile residual stress field around a NMP due to the elastic incompatibility as well as, the thermal expansion mismatch with the matrix material $[13,14]$. The effect of stress level on the small crack growth behavior also appears to be significant (Fig. 4(b)) in this material. These small crack growth effects need to be accounted for, and will play an important role in the proposed probabilistic life-prediction model.

The role of small crack growth in the separation of lifetimes was first studied with the help of deterministic calculations. For this, the crack growth lifetime bounds were calculated by considering the upper and the lower bound on the small crack growth behavior in conjunction with the maximum and the minimum crack initiation size respectively. The life-limiting behavior corresponded to crack initiation from a NMP. However, due to the small frequency of occurrence of the NMP-initiated mechanism, only one small crack growth curve was available in that crack initiation mode (Fig. 4(a)), which was taken as the upper bound of 
the small crack growth behavior for the calculation purposes. The lower limit of the NMP-initiated small crack growth behavior was assumed to correspond to the upper-bound crack growth curve of the pore-initiated mechanism (Fig. 4(a)). This is illustrated in Fig. 5. As shown, multiple power-law fitted segments (labeled as S1 to S3) were employed to represent each crack growth curve to obtain the crack growth rate $(\mathrm{da} / \mathrm{dN})-\Delta \mathrm{K}$ relationship for the lifetime calculations. Similarly, due to the small number of small crack growth experiments at $1200 \mathrm{MPa}$, the upper-bound small crack growth curve from the pore-initiated mechanism at $1150 \mathrm{MPa}$ was also taken as the lower-limit curve at $1200 \mathrm{MPa}$. These approximations were considered suitable in capturing the shift to higher small crack growth rates with stress level, and for crack initiation from a NMP. Additional small crack growth data under these specific conditions will certainly improve the accuracy of the predictions. For crack initiation size calculation, two schemes were used. In these, the crack depth, $a$, was taken as (i) the radius of the equivalent semicircle with the same area as the crack initiating feature, and (ii) the radius of the semicircle circumscribing the crack initiation feature. The range in the NMPrelated crack initiation sizes, which were measured in the fracture surfaces, was used in the calculations at all stress levels.

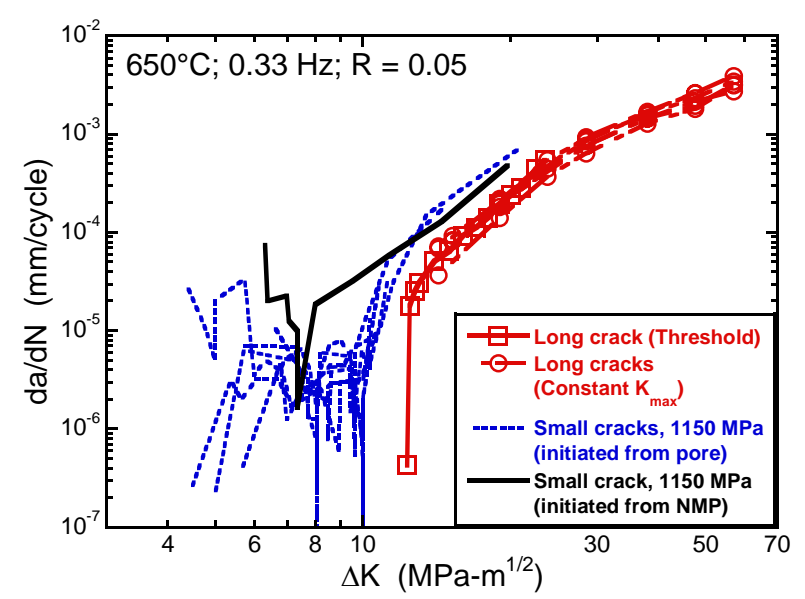

(a)

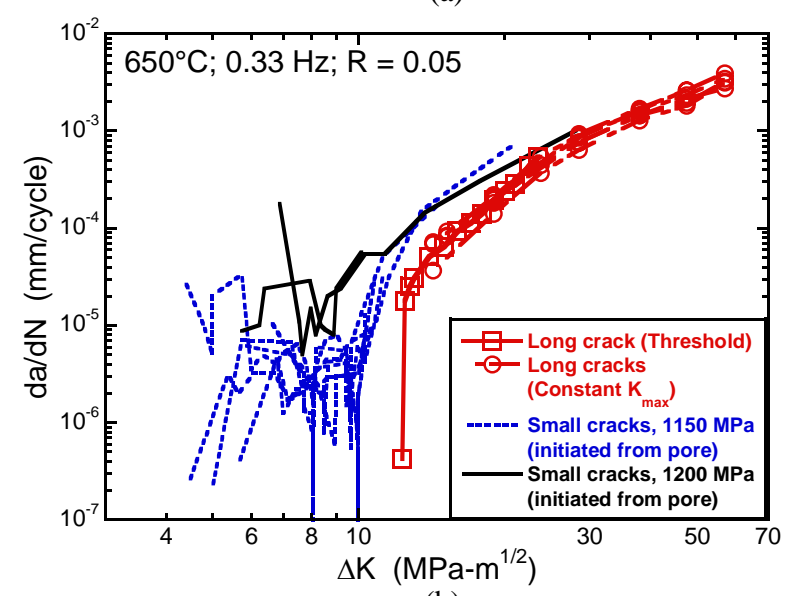

(b)

Figure 4. Small crack growth behavior of IN100; (a) Effect of crack initiation from NMP vs. pore and (b) Effect of stress level on the growth rates.

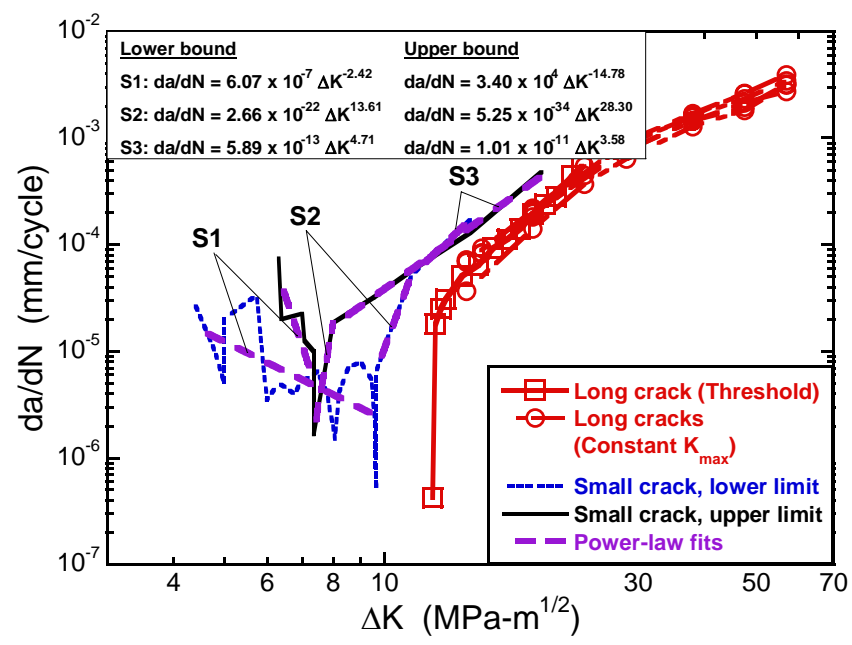

Figure 5. Small crack growth bounds and the fitted power-law segments used in the deterministic calculations at 1150 and 1100 MPa.

The role of small crack growth in the separation of mean and the life-limiting behavior is illustrated in Fig. 6. The figure shows, that the calculated crack growth bounds reasonably represent the range in the life-limiting behavior. The mean-lifetime response, on the other hand, separates from the crack-growth-controlled behavior with decreasing stress level. The lifetime probability density can therefore, be modeled as a superposition of the crack growth behavior upon the mean-lifetime behavior. The effect of microstructure and loading variables on the lifetime variability can then be evaluated by separating their effects on the crack growth lifetime and the mean lifetime, as illustrated in a forthcoming section.

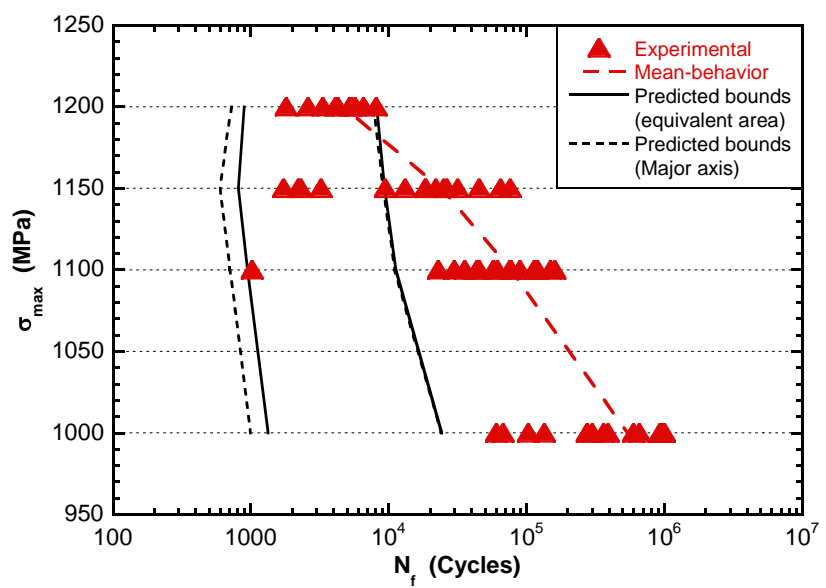

Figure 6. Role of small crack growth in the separation of meanbehavior from the life-limiting response.

\section{Effect of dwell loading on the mean vs. life-limiting behavior}

From the above discussion, the effect of dwell loading on the lifetime variability can be described in terms of separate effects on the crack growth lifetime and the mean-dominating behavior. The small crack growth data, obtained at 1150 and $1100 \mathrm{MPa}$, with 6 second dwell at the peak load is shown in Fig. 7. The figure indicates that, while the long-crack regime was not significantly 
influenced by the dwell loading, it had a significant effect on the small crack growth rates when compared at the same stress level. Also, there was a strong effect of stress level on the small crack growth regime under dwell loading. As indicated previously, in the proposed life-prediction approach, characterizing these effects is vital in determining the life-limiting behavior and the probabilistic lifetime limit.

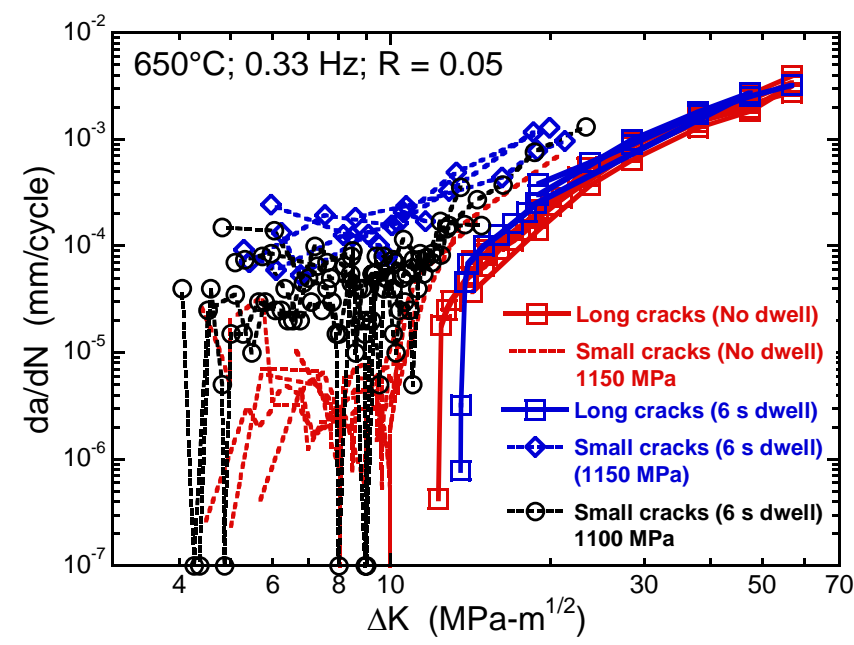

Figure 7. The effect of dwell time on the small crack growth regime in IN100.

The mean vs. life-limiting behavior under dwell loading is presented in Fig. 8. The figure shows that the 6 second peak dwell produced a significant reduction in the mean-lifetime (in terms of cycles) but a much weaker effect on the life-limiting behavior. The calculated crack growth bounds, based on the small crack growth variability at $1100 \mathrm{MPa}$ (Fig. 7) and the range in the crack initiation sizes, are superimposed in the figure and, once again, illustrates that the life-limiting fatigue response is reasonably captured by the range in the crack growth lifetime. Comparing the two cases, i.e., with and without dwell, it appears that the separation of mean from the life-limiting behavior is related to this control of the life-limiting response by the crack growth regime, which promoted a smaller degree of change in the lifelimiting behavior with respect to both the stress-level and the dwell-time. The mean-lifetime, on the other hand, showed a significantly stronger sensitivity to these variables.

\section{Calculation of the lifetime probability density}

Calculation Procedure. The different rates of response of the crack-growth-controlled behavior and the mean-dominating behavior, as discussed above, may explain the increased separation/overlap between the two as a function of stress level and dwell time, thereby affecting the total lifetime variability. The lifetime probability density can therefore, be represented as a superposition of the crack growth probability density function (PDF) and a mean-dominating PDF [10]:

$$
f_{t}(x)=p_{l} f_{l}(x)+p_{m} f_{m}(x)
$$

where, $f_{t}(x), f_{l}(x)$, and $f_{m}(x)$ are the total lifetime, the life-limiting, and the mean-dominating probability densities respectively. The weighting parameters, $p_{l}$ and $p_{m}$, are the probability of occurrences of the crack-growth-controlled and the mean- dominating mechanism respectively. It can be shown that the lower-tail of the above bimodal probability density, which is most pertinent in the calculation of the probabilistic lifetime-limit, is strongly governed by $f_{l}(x)$ and does not show substantial change within reasonable range of values of $p_{l}$ and $p_{m}$ [10]. Furthermore, the probabilistic limit, for e.g., the B0.1 lifetime, can be shown to be bounded by the B0.1 lifetime obtained from the crack growth density [10] (i.e., $p_{m} \rightarrow 0$ ). In the present case, the values of $p_{l}$ and $p_{m}$ were derived from the $\mathrm{S}-\mathrm{N}$ experiments.

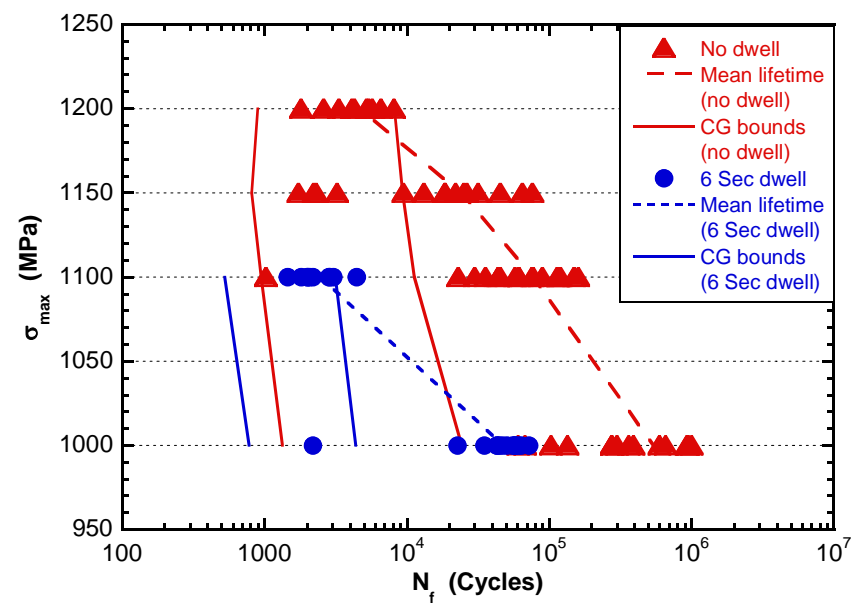

Figure 8. The role of small crack growth in the effect of dwell time on the mean vs. life-limiting fatigue response.

The life-limiting density, $f_{l}(x)$, was simulated by using the crack initiation size distribution [7] and the small crack growth data. The crack initiation size was measured on the fatigue fracture surfaces and was modeled by the lognormal probability density function. This is shown in Fig. 9(a). The small crack growth data were fitted by multiple power-law (Paris) segments [15] such that:

$\frac{d a}{d N}=e^{C} \Delta K^{m}$

The Paris-type coefficient and exponent, $C$ and $m$, were treated as random variables and were modeled by the normal density function [16]. The parameters of $C$ and $m$ were derived by the Maximum Likelihood Estimate (MLE) method using the available small crack growth data. It is also recognized that the variables, $C$ and $m$ are strongly negatively correlated $[15,16]$ and this correlation was invoked in the simulation of lifetimes. The joint $\mathrm{PDF}$ of $C$ and $m$ for the lower- $\Delta \mathrm{K}$ small-crack growth segment in the no-dwell case is presented in Fig. 9 (b).

The CDFs associated with the simulated life-limiting densities, $f_{l}(x)$, are shown in Figs. 10 and 11 for the 0 second and the 6 second peak dwell cases, respectively. The crack-growthcontrolled densities show reasonable correspondence with the observed distribution in lifetime at the higher stress levels, as in Figs. 10(a) and 11 (a) (due to convergence of the mean and the life-limiting behavior) but describe the life-limiting response at the lower stress levels, as illustrated in Figs. 10(b and c) and 11(b).

The mean-dominating density, $f_{m}(x)$, can be estimated by running a relatively small number of $\mathrm{S}-\mathrm{N}$ experiments under the 
conditions of interest. This is illustrated in Fig. 12. Here, 4 tests were randomly selected at each stress level and for the no-dwell and the peak dwell condition. The parameters of $f_{m}(x)$ were then determined by the MLE method assuming the lognormal model.

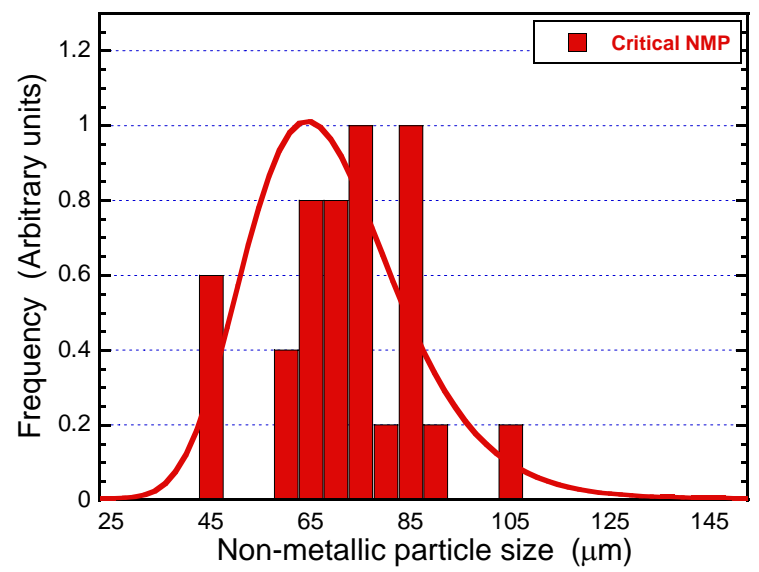

(a)

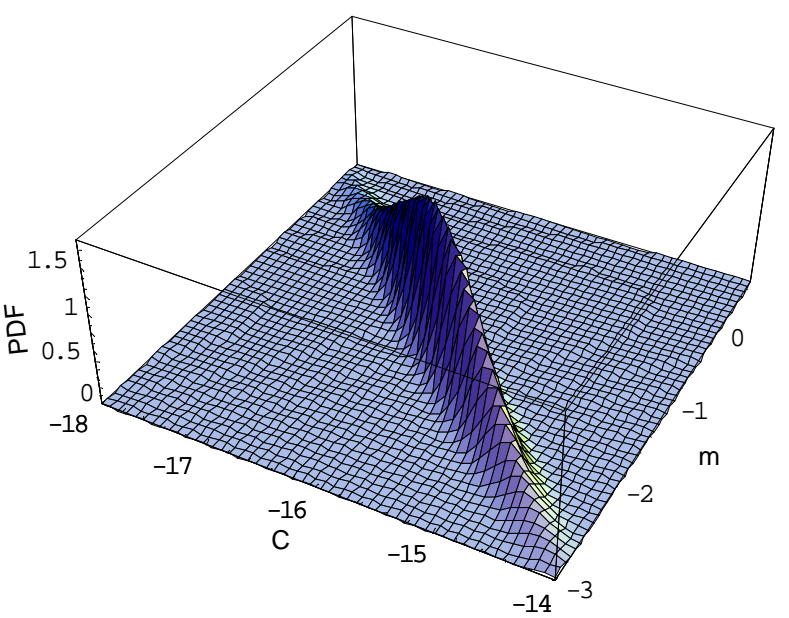

(b)

Figure 9. Examples of input to the simulation of the life-limiting probability density, $f_{l}(x)$; (a) The crack initiation size distribution for the NMP initiated cracks in terms of the diameter of the equivalent circle, and (b) The joint PDF of the small crack growth variables, $\mathrm{C}$ and $\mathrm{m}$ for the case of 0 dwell time.

Predicted Lifetime Densities. The predicted lifetime densities, calculated according to Eqn. (1), are shown in Figs. 13 (a) and (b) for the two loading conditions. The conventional description of fatigue variability, where the lower tail is considered as an extrapolation of deviations from the mean behavior, is also plotted in these figures. The B0.1 lifetimes obtained by the two approaches have been indicated by vertical lines and the experimental points are represented by open symbols. The results show that the suggested lifetime modeling provides a more accurate representation of the effect of stress level and dwell time on fatigue variability. Especially, the predictions of the lower tail and the B0.1 lifetimes are more consistent with respect to these variables and almost independent of the number of $\mathrm{S}-\mathrm{N}$ tests therefore, significantly decreasing the uncertainty in the probabilistic lifetime limit. For example, although the total

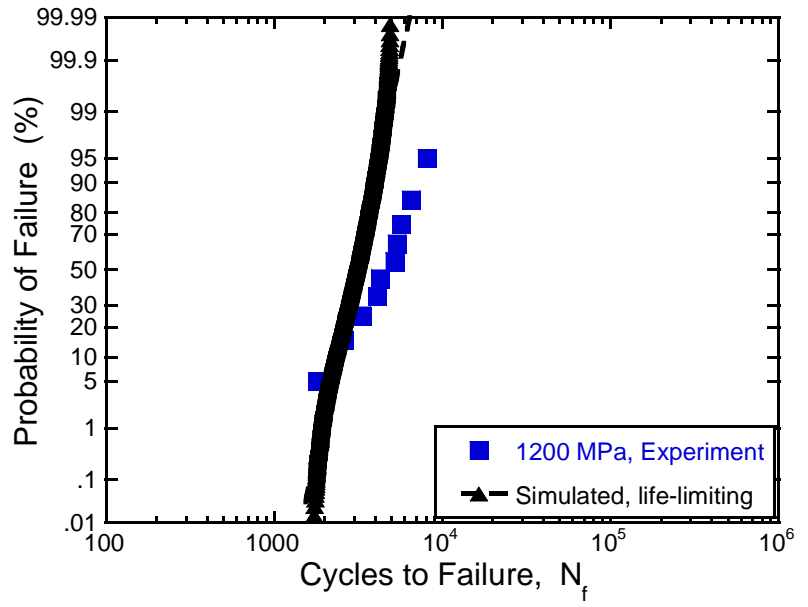

(a)

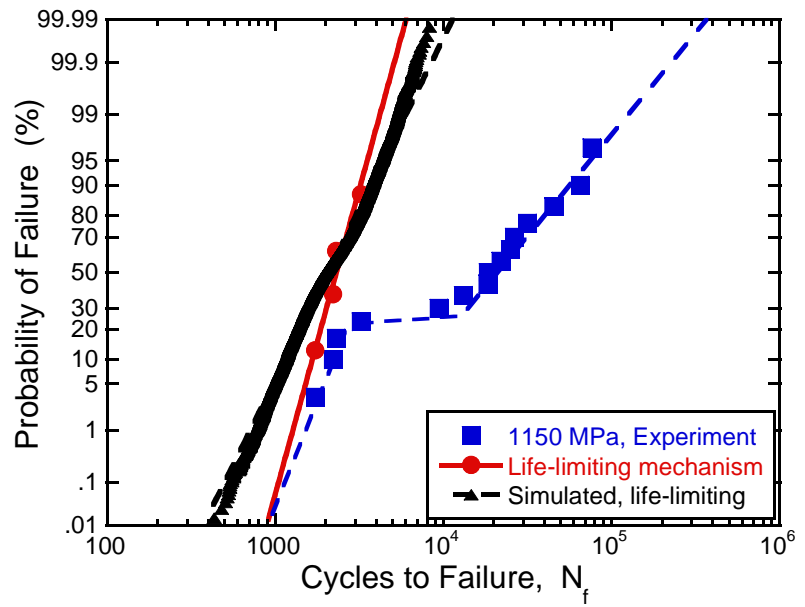

(b)

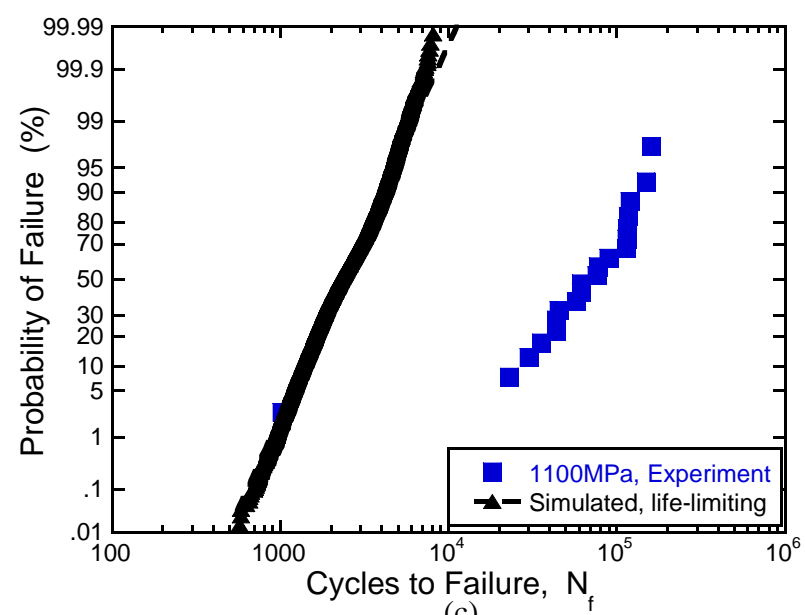

Figure 10. The CDFs of the calculated life-limiting probability densities for the case of no-dwell; (a) $\sigma_{\max }=1200 \mathrm{MPa}$, (b) $\sigma_{\max }=$ $1150 \mathrm{MPa}$, and (c) $\sigma_{\max }=1100 \mathrm{MPa}$.

variability (and mean-lifetime) increased with decreasing stress level and dwell time, this is attributed to the separate degree of effects of these variables on the small crack growth regime and the mean-lifetime behavior. Consequently, the lower-tail and the B0.1 lifetime are not entirely dependent on the mean-behavior or 
the number of data points, producing, perhaps, a physically more accurate trend in the probabilistic lifetime limits (Fig. 13).

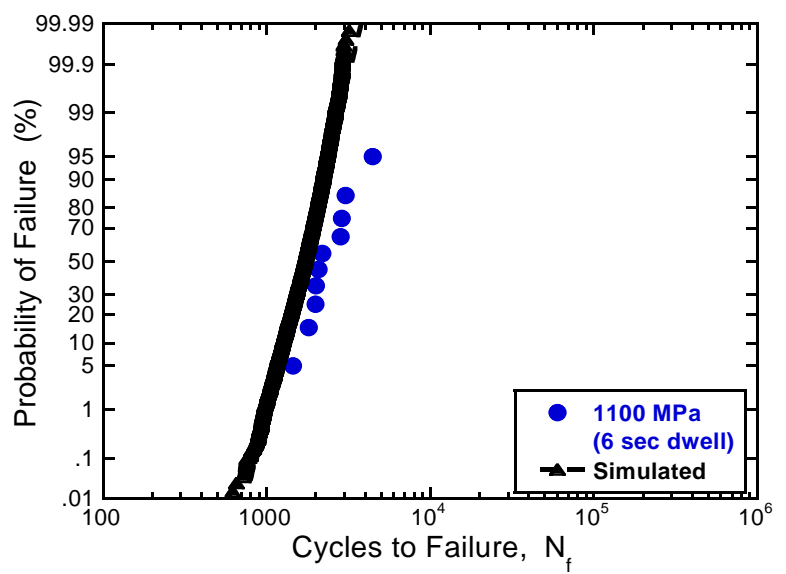

(a)

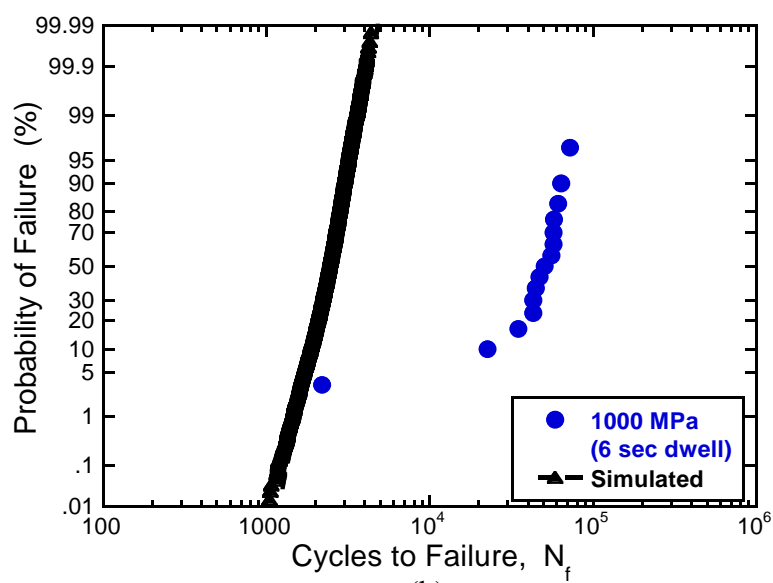

(b)

Figure 11. The CDFs associated with the calculated life-limiting densities for the case of 6 second peak dwell; (a) $\sigma_{\max }=1100$ $\mathrm{MPa}$ and (b) $\sigma_{\max }=1000 \mathrm{MPa}$.

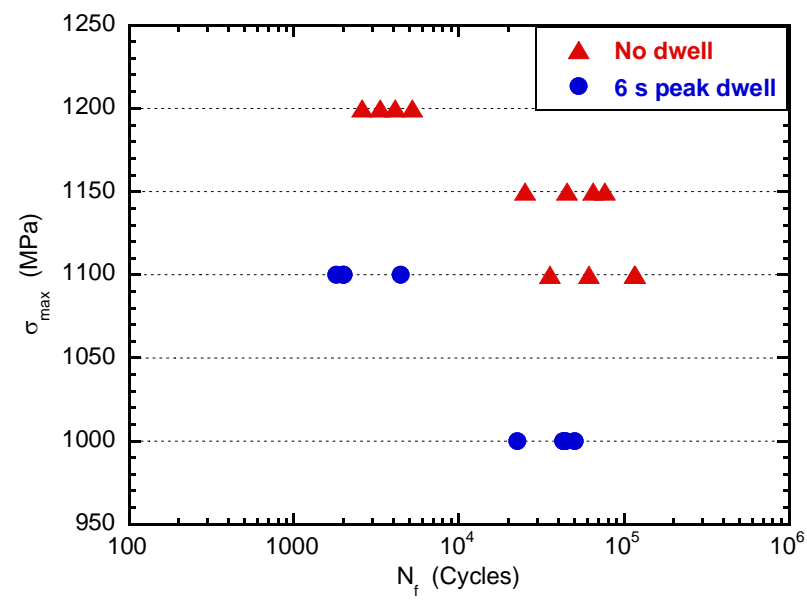

Figure 12. Input data (4 random tests at each stress level) for obtaining the mean-lifetime-dominating density, $f_{m}(x)$.

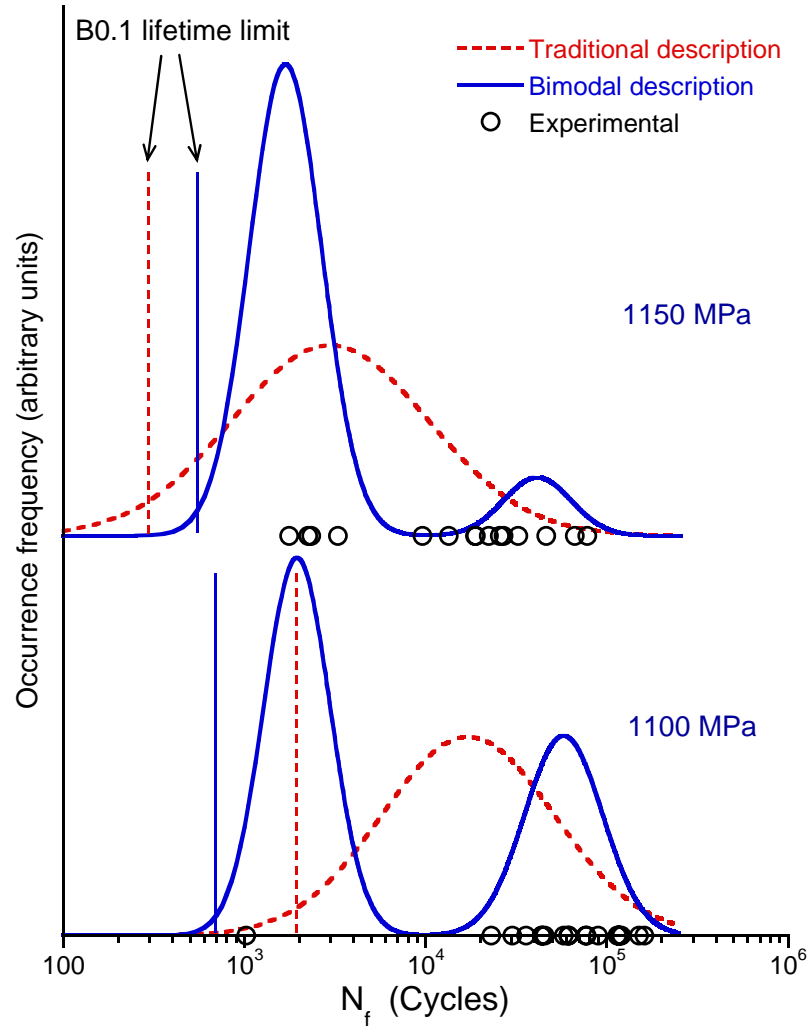

(a)
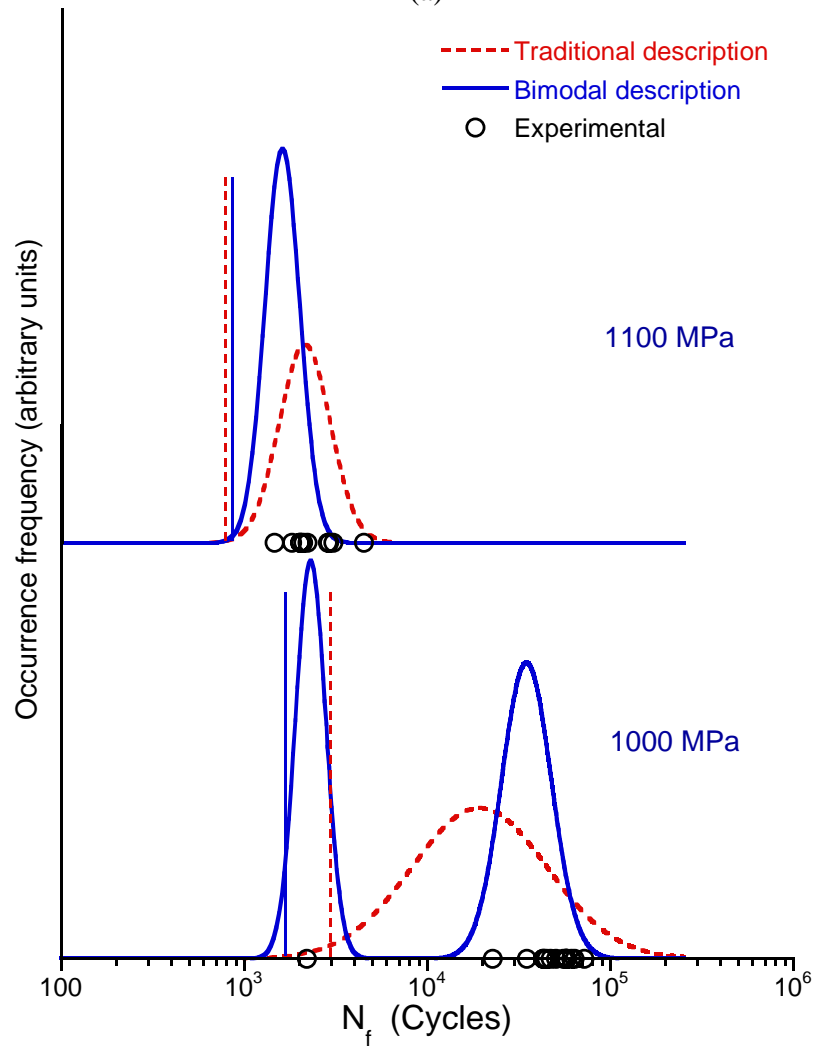

(b)

Figure 13. Predicted lifetime probability densities and the B0.1 lifetimes based on the proposed fatigue variability description; (a) no-dwell and (b) 6 second peak dwell. 
As indicated above, a distinct attribute of the suggested method is that, the predictions of the B0.1 lifetimes are not heavily dependent on the time consuming, and often expensive S-N fatigue experiments. Notably, the predicted CDFs account for the solitary life-limiting failure at $1100 \mathrm{MPa}$ with no-dwell (Fig. 10(c)) and at $1000 \mathrm{MPa}$ under dwell loading (Fig. 11(b)), which, due to the small probability of occurrence, otherwise requires a relatively large number of total lifetime experiments to capture. Nonetheless, the key to the method is the recognition that the lifetime variability is a result of separate responses of the crackgrowth-controlled behavior and the mean-dominating behavior with respect to both the intrinsic and the extrinsic variables. Therefore, merely a large number of experiments may still not be sufficient in accurate prediction of the probabilistic lifetime limits without the paradigm shift in the description of fatigue variability.

\section{Conclusions}

Based on this study, the following concluding points can be made: The effect of stress level and dwell time on the fatigue variability and the probabilistic lifetime limit can be accurately predicted by considering separate responses of the crack-growth-controlled life-limiting behavior and the mean-lifetime behavior to these variables.

(ii) The lifetime probability density can therefore, be modeled as a superposition of the crack growth lifetime density and the mean-dominating density.

(iii) The B0.1 lifetimes predicted by this approach show a more consistent trend with respect to stress level and dwell time independently of the number of S-N fatigue experiments therefore, significantly decreasing the uncertainty in these probabilistic limits.

\section{Acknowledgements}

This work was performed at the Air Force Research Laboratory, Materials and Manufacturing Directorate, Wright-Patterson Air Force Base, OH. The partial financial support of the Defense Advanced Research Project Agency (DARPA) under DARPA orders M978, Q588, P699, and S271 with Dr. Leo Christodoulou as the program manager is gratefully acknowledged. Essential support was also provided by the Air Force Office of Scientific Research (AFOSR) with Dr. Victor Giurgiutiu as the program manager. We acknowledge Mr. Phil Buskohl and Ms. Lindsey Selegue for their assistance with the replication-based small-crack growth experiments.

\section{References}

1. L. Christodoulou and J. M. Larsen, JOM, p. 15, March, 2004.

2. J. W. Lincoln, Journal of Aircraft, Vol. 22, 1985.

3. J. N. Yang and W. J. Trapp, AIAA Journal, Vol. 12, pp. 1623-1630, 1974.

4. R. Tryon and A. Dey, J. Aerospace Engng., p.120, 2001.

5. K. S. Chan and M. P. Enright, Journal of Engineering for Gas Turbines and Power, Vol. 124, pp. 889-885, 2006.

6. A. de Bussac, and J. C. Lautridou, Fatigue Fract. Engng. Mater. Struct., Vol. 16, p. 861, 1993.

7. S. K. Jha, M. J. Caton, and J. M. Larsen, Materials Science and Engineering A, Vol. A468-470, pp. 23-32, 2007.
8. S. K. Jha, J. M. Larsen, and A. H. Rosenberger, Acta Materialia, Vol. 53, pp. 1293-1304, 2005.

9. S. K. Jha and J. M. Larsen, in Very High Cycle Fatigue, VHCF-4, 2007.

10. S. K. Jha, J. M. Larsen, and A. H. Rosenberger, in review, Engineering Fracture Mechanics.

11. R. G. Forman, and V. Shivakumar, in Fracture Mechanics: $17^{\text {th }}$ Volume, ASTM STP 905, J. H. Underwood, R. Chait, C. W. Smith, D. P. Wilhem, W. A. Andrews, and J. C. Newman, Eds., ASTM, Philadelphia, pp. 59-74, 1986.

12. J. C. Newman, Jr. and I. S. Raju, Engineering Fracture Mechanics, Vol. 15, pp. 185-192, 1981.

13. M. M. Shenoy, R. S. Kumar, and D. L. McDowell, Int. J. Fatigue, Vol. 27, p. 113, 2005.

14. R. Bullough and L. C. Davis, Acta Materialia, Vol. 43, p. 2737, 1995.

15. J. Luo, and P. Bowen, Acta Materialia, Vol. 51, pp. 35373550, 2003.

16. C. G. Annis, Jr., in Probabilistic Aspects of Life Prediction, ASTM-STP 1450, W. J. Johnson and B. M. Hillberry, Eds., ASTM International, West Conshohocken, PA, 2003. 